\title{
Article
}

\section{Differential operators and Narayana numbers}

\section{Jie Xiong ${ }^{1}$ and Qi Fang ${ }^{2, *}$}

1 School of Mathematics,Northeastern University, Shenyang 110004, P. R. China.; xiongjiepapers@163.com

2 School of Mathematics, Northeastern University, Shenyang 110004, P. R. China.

* Correspondence: qifangpapers@stumail.neu.edu.cn

Received: 1 January 2020; Accepted: 10 March 2020; Published: 30 March 2020.

Abstract: In this paper, we establish a connection between differential operators and Narayana numbers of both kinds, as well as a kind of numbers related to central binomial coefficients studied by Sulanke (Electron. J. Combin. 7 (2000), R40).

Keywords: Narayana numbers, recurrence relations, differential operators.

MSC: 05A05, 26A33.

\section{Introduction}

I

$\mathrm{t}$ is well known that the central binomial coefficients have the following expressions;

$$
\left(\begin{array}{c}
2 n \\
n
\end{array}\right)=\sum_{k=0}^{n}\left(\begin{array}{l}
n \\
k
\end{array}\right)^{2},\left(\begin{array}{c}
2 n+1 \\
n
\end{array}\right)=\sum_{k=0}^{n}\left(\begin{array}{l}
n \\
k
\end{array}\right)\left(\begin{array}{c}
n+1 \\
k
\end{array}\right)
$$

For $0 \leq k \leq n$, the Narayana numbers of types $A$ are defined as;

$$
N(n, k)=\frac{1}{n}\left(\begin{array}{c}
n \\
k+1
\end{array}\right)\left(\begin{array}{l}
n \\
k
\end{array}\right) .
$$

Let $N_{n}(x)=\sum_{k=0}^{n-1} N(n, k) x^{k}$ be the Narayana polynomials of types $A$ (see [1]). It is well known that $N_{n}(x)$ is the rank-generating function of the lattice of non-crossing partition lattice with cardinality $\frac{1}{n+1}\left(\begin{array}{c}2 n \\ n\end{array}\right)$ (see [2]). Hence the Catalan numbers have the following expression;

$$
\frac{1}{n+1}\left(\begin{array}{c}
2 n \\
n
\end{array}\right)=\sum_{k=0}^{n-1} \frac{1}{n}\left(\begin{array}{c}
n \\
k+1
\end{array}\right)\left(\begin{array}{l}
n \\
k
\end{array}\right)
$$

The Narayana numbers of type $B$ are given as;

$$
M(n, k)=\left(\begin{array}{l}
n \\
k
\end{array}\right)^{2} .
$$

Let $M_{n}(x)=\sum_{k=0}^{n} M(n, k) x^{k}$. Reiner [2] showed that $M_{n}(x)$ is the rank-generating function of a ranked self-dual lattice with the cardinality $\left(\begin{array}{c}2 n \\ n\end{array}\right)$.

Let $P(n, k)=\left(\begin{array}{c}n \\ k\end{array}\right)\left(\begin{array}{c}n+1 \\ k\end{array}\right)$, and $S=\mathbb{P} \times \mathbb{P}$. According to [3, Proposition 1], $P(n, k)$ is the number of paths in $A_{1}(n+1)$ having $k+1$ steps, where $A_{1}(n)$ is the set of all lattice paths running from $(0 ;-1)$ to $(n ; n)$ that use the steps in $S$ and that remain strictly above the line $y=-1$ except initially.

The numbers $N(n, k), M(n, k)$ and $P(n, k)$ have been extensively studied. The readers are referred to [4] for details. In [5], Daboul et al., reveals that

$$
\frac{d^{n}}{d x^{n}}\left(e^{1 / x}\right)=(-1)^{n} e^{1 / x} \sum_{k=1}^{n}\left(\begin{array}{l}
n \\
k
\end{array}\right)\left(\begin{array}{l}
n-1 \\
k-1
\end{array}\right)(n-k) ! x^{-n-k}
$$


where the $\left(\begin{array}{l}n \\ k\end{array}\right)\left(\begin{array}{l}n-1 \\ k-1\end{array}\right)(n-k)$ ! are the Lah numbers. Motivated by this result, in this paper we show that the numbers $M(n, k), N(n, k)$ and $P(n, k)$ can be generated by higher-order derivative of functions of $e^{x}$. As an application, we obtain new recurrence relations for these classical combinatorial numbers.

\section{Differential operators and Narayana polynomials}

Let $P_{n}(x)=\sum_{k=0}^{n} P(n, k) x^{n-k}, Q_{n}(x)=\sum_{k=0}^{n} P(n, k) x^{k}$, then $Q_{n}(x)=x^{n} P_{n}(1 / x)$. The first few $N_{n}(x), M_{n}(x)$ and $P_{n}(x)$ are listed as follows;

$$
\begin{aligned}
& N_{1}(x)=1, N_{2}(x)=1+x, N_{3}(x)=1+3 x+x^{2}, N_{4}(x)=1+6 x+6 x^{2}+x^{3}, \\
& M_{1}(x)=1+x, M_{2}(x)=1+4 x+x^{2}, M_{3}(x)=1+9 x+9 x^{2}+x^{3}, \\
& P_{1}(x)=2+x, P_{2}(x)=3+6 x+x^{2}, P_{3}(x)=4+18 x+12 x^{2}+x^{3} .
\end{aligned}
$$

We define $\bar{N}(n, k)=(n+1) ! n ! N(n, k)$ and $\bar{M}(n, k)=n !^{2} M(n, k)$. By using the explicit formulas of $\bar{N}(n, k)$ and $\bar{M}(n, k)$, it is routine to verify the following lemma.

Lemma 1. For $0 \leq k \leq n+1$, we have

$$
\begin{aligned}
\bar{N}(n+1, k)= & \left((n+1)(n+2)+2 n k+k^{2}+3 k\right) \bar{N}(n, k)+\left(4 n+2 n^{2}-2\left(k^{2}-1\right)\right) \bar{N}(n, k-1) \\
& +(n(n-1)-(k-2)(2 n-k+1)) \bar{N}(n, k-2), \\
\bar{M}(n+1, k)= & \left((n+1)^{2}+2(n+1) k+k^{2}\right) \bar{M}(n, k)+\left(1+4 n+2 n^{2}-2 k(k-1)\right) \bar{M}(n, k-1) \\
& +\left(n^{2}-(2 n+2-k)(k-2)\right) \bar{M}(n, k-2),
\end{aligned}
$$

with initial conditions $\bar{N}(0,0)=\bar{M}(0,0)=1$ and $\bar{N}(0, k)=\bar{M}(0, k)=0$ for $k \neq 0$.

In the following discussion, let $D=\frac{d}{d x}$.

Theorem 1. For $n \geq 1$, we have

$$
\begin{aligned}
\left(D e^{x} D\right)^{n}\left(\frac{1}{1-e^{x}}\right) & =\frac{n !(n+1) ! e^{(n+1) x} N_{n}\left(e^{x}\right)}{\left(1-e^{x}\right)^{2 n+1}} \\
\left(e^{x} D^{2}\right)^{n}\left(\frac{1}{1-e^{x}}\right) & =\frac{n !^{2} e^{(n+1) x} M_{n}\left(e^{x}\right)}{\left(1-e^{x}\right)^{2 n+1}} \\
\left(D^{2} e^{x}\right)^{n}\left(\frac{1}{1-e^{x}}\right) & =\frac{n !^{2} e^{n x} M_{n}\left(e^{x}\right)}{\left(1-e^{x}\right)^{2 n+1}}
\end{aligned}
$$

Proof. Note that

$$
\begin{aligned}
\left(D e^{x} D\right)\left(\frac{1}{1-e^{x}}\right) & =\frac{2 e^{2 x}}{\left(1-e^{x}\right)^{3}} \\
\left(D e^{x} D\right)^{2}\left(\frac{1}{1-e^{x}}\right) & =\frac{12 e^{3 x}\left(1+e^{x}\right)}{\left(1-e^{x}\right)^{5}} \\
\left(D e^{x} D\right)^{3}\left(\frac{1}{1-e^{x}}\right) & =\frac{144 e^{4 x}\left(1+3 e^{x}+e^{2 x}\right)}{\left(1-e^{x}\right)^{7}}
\end{aligned}
$$

Hence the formula (1) holds for $n=1,2,3$. Assume that the result holds for $n$, where $n \geq 3$. Let $\bar{N}_{n}(x)=$ $\sum_{k=0}^{n-1} \bar{N}(n, k) x^{k}$. Note that

$$
\left(D e^{x} D\right)^{n+1}\left(\frac{1}{1-e^{x}}\right)=\left(D e^{x} D\right)\left(\frac{e^{(n+1) x} \bar{N}_{n}\left(e^{x}\right)}{\left(1-e^{x}\right)^{2 n+1}}\right) .
$$

It follows that

$\bar{N}_{n+1}(x)=\left((n+1)(n+2)+\left(4 n+2 n^{2}\right) x+n(n-1) x^{2}\right) \bar{N}_{n}(x)+\left(4 x-6 x^{2}+2 x^{3}+2 n x\left(1-x^{2}\right)\right) D\left(\bar{N}_{n}(x)\right)$ $+x^{2}(1-x)^{2} D^{2}\left(\bar{N}_{n}(x)\right)$. 
Equating the coefficients of $x^{k}$ in both sides, we immediately get the recurrence relation of $\bar{N}(n, k)$ given in Lemma 1. Therefore, the result holds for $n+1$.

Similarly, note that

$$
\begin{aligned}
\left(e^{x} D^{2}\right)\left(\frac{1}{1-e^{x}}\right) & =\frac{e^{2 x}\left(1+e^{x}\right)}{\left(1-e^{x}\right)^{3}} \\
\left(e^{x} D^{2}\right)^{2}\left(\frac{1}{1-e^{x}}\right) & =\frac{4 e^{3 x}\left(1+4 e^{x}+e^{2 x}\right)}{\left(1-e^{x}\right)^{5}} \\
\left(e^{x} D^{2}\right)^{3}\left(\frac{1}{1-e^{x}}\right) & =\frac{36 e^{4 x}\left(1+9 e^{x}+9 e^{2 x}+e^{3 x}\right)}{\left(1-e^{x}\right)^{7}} .
\end{aligned}
$$

Hence the formula (2) holds for $n=1,2,3$. Assume it holds for $n$, where $n \geq 3$. Let $\bar{M}_{n}(x)=$ $\sum_{k=0}^{n} \bar{M}(n, k) x^{k}$. Note that

$$
\left(e^{x} D^{2}\right)^{n+1}\left(\frac{1}{1-e^{x}}\right)=\left(e^{x} D^{2}\right)\left(\frac{e^{(n+1) x} \bar{M}_{n}\left(e^{x}\right)}{\left(1-e^{x}\right)^{2 n+1}}\right)
$$

It follows that

$\bar{M}_{n+1}(x)=\left(1+x+n^{2}(1+x)^{2}+n(2+4 x)\right) \bar{M}_{n}(x)+\left(3 x-4 x^{2}+x^{3}+2 n x\left(1-x^{2}\right)\right) D\left(\bar{M}_{n}(x)\right)$ $+x^{2}(1-x)^{2} D^{2}\left(\bar{M}_{n}(x)\right)$.

Equating the coefficients of $x^{k}$ in both sides, we immediately get the recurrence relation of $\bar{M}(n, k)$ given in Lemma 1. Therefore, the result holds for $n+1$. Along the same lines, it is routine to derive (3). This completes the proof.

Note that $P(n, n-k)=\left(\begin{array}{c}n \\ n-k\end{array}\right)\left(\begin{array}{c}n+1 \\ n-k\end{array}\right)$, then $P(n+1, n+1-k)=\left(\begin{array}{c}n+1 \\ n+1-k\end{array}\right)\left(\begin{array}{c}n+2 \\ n+1-k\end{array}\right)$.

It is easy to verify the following lemma;

Lemma 2. For $0 \leq k \leq n+1$, we have $(n+1)(n+2) P(n+1, n+1-k)=\left[(n+2)^{2}+(2 n+5) k+k(k-\right.$ 1)] $P(n, n-k)+\left[2\left(n^{2}+3 n+1\right)-6(k-1)-2(k-1)(k-2)\right] P(n, n-k+1)+\left[n^{2}-(2 n-1)(k-2)+(k-\right.$ 2) $(k-3)] P(n, n-k+2)$.

Theorem 2. For $n \geq 1$, we have

$$
\begin{aligned}
& \left(D^{2} e^{x}\right)^{n} \frac{e^{x}}{\left(1-e^{x}\right)^{2}}=\frac{n !(n+1) ! e^{(n+1) x} P_{n}\left(e^{x}\right)}{\left(1-e^{x}\right)^{2 n+2}} \\
& \left(D e^{x} D\right)^{n} \frac{e^{x}}{\left(1-e^{x}\right)^{2}}=\frac{n !(n+1) ! e^{(n+1) x} Q_{n}\left(e^{x}\right)}{\left(1-e^{x}\right)^{2 n+2}} .
\end{aligned}
$$

Proof. Note that

$$
\begin{gathered}
\left(D^{2} e^{x}\right) \frac{e^{x}}{\left(1-e^{x}\right)^{2}}=\frac{2 e^{2 x}\left(2+e^{x}\right)}{\left(1-e^{x}\right)^{4}}, \\
\left(D^{2} e^{x}\right)^{2} \frac{e^{x}}{\left(1-e^{x}\right)^{2}}=\frac{12 e^{3 x}\left(3+6 e^{x}+e^{2 x}\right)}{\left(1-e^{x}\right)^{6}} .
\end{gathered}
$$

Hence the result holds for $n=1,2$. Assume that the result holds for $n$. Then from (4), we get the recurrence relation

$(n+1)(n+2) P_{n+1}(x)=\left[n^{2} x^{2}+(2+n)^{2}+2 x\left(1+3 n+n^{2}\right)\right] P_{n}(x)+x(1-x)[(2 n-1) x+2 n+5] P_{n}^{\prime}(x)+x^{2}(1-$ $x)^{2} P_{n}^{\prime \prime}(x)$.

Equating the coefficients of $x^{k}$ in both sides, we get the recurrence relation of the numbers $P(n, n-k)$, which is given in Lemma 2, as desired. Along the same lines, one can derive (5). This completes the proof.

By a change of variable $y=e^{x}$, we end our paper by giving a corollary;

Corollary 1. For $n \geq 1$, let $D_{y}=\frac{d}{d y}$, we have 
1. $\left(y D_{y} y^{2} D_{y}\right)^{n}\left(\frac{1}{1-y}\right)=\frac{n !(n+1) ! y^{n+1} N_{n}(y)}{(1-y)^{2 n+1}}$,

2. $\left(y^{2} D_{y} y D_{y}\right)^{n}\left(\frac{1}{1-y}\right)=\frac{n !^{2} y^{(n+1)} M_{n}(y)}{(1-y)^{2 n+1}}$,

3. $\left(y D_{y} y D_{y} y\right)^{n}\left(\frac{1}{1-y}\right)=\frac{n !^{2} y^{n} M_{n}(y)}{(1-y)^{2 n+1}}$,

4. $\left(y D_{y} y D_{y} y\right)^{n} \frac{y}{(1-y)^{2}}=\frac{n !(n+1) ! y^{(n+1)} P_{n}(y)}{(1-y)^{2 n+2}}$,

5. $\left(y D_{y} y^{2} D_{y}\right)^{n} \frac{y}{(1-y)^{2}}=\frac{n !(n+1) ! y^{(n+1)} Q_{n}(y)}{(1-y)^{2 n+2}}$.

Proof. It's not hard to verify the equations hold when $n=1,2$

$$
\begin{gathered}
\left(y D_{y} y^{2} D_{y}\right)\left(\frac{1}{1-y}\right)=\frac{2 y^{2}}{(1-y)^{3}}, \\
\left(y D_{y} y^{2} D_{y}\right)^{2}\left(\frac{1}{1-y}\right)=\frac{12 y^{3}(1+y)}{(1-y)^{5}} .
\end{gathered}
$$

Assume the result holds for $m$, where $m \geq 3$. Setting $y=e^{x}$, we get

$$
\begin{aligned}
\left(y D_{y} y^{2} D_{y}\right)\left(y D_{y} y^{2} D_{y}\right)^{m}\left(\frac{1}{1-y}\right)= & \left(e^{x} D_{y} e^{2 x} D_{y}\right) \frac{m !(m+1) ! y^{n+1} N_{m}(y)}{(1-y)^{2 m+1}} \\
= & \left(D e^{x} D\right) \frac{m !(m+1) ! e^{(m+1) x} N_{m}\left(e^{x}\right)}{\left(1-e^{x}\right)^{2 m+1}} \\
= & \frac{(m+1) !(m+2) ! e^{(m+2) x} N_{m+1}\left(e^{x}\right)}{\left(1-e^{x}\right)^{2 m+3}} \\
& =\frac{(m+1) !(m+2) ! y^{m+1} N_{m+1}(y)}{(1-y)^{2 m+3}} .
\end{aligned}
$$

Along the same lines, we can get the other statements. This completes the proof.

Acknowledgments: The authors appreciate the careful review, corrections and helpful suggestions to this paper made by the referee.

Author Contributions: All authors contributed equally to the writing of this paper. All authors read and approved the final manuscript.

Conflicts of Interest: "The authors declare no conflict of interest."

\section{References}

[1] Bonin, J., Shapiro, L., \& Simion, R. (1993). Some q-analogues of the Schröder numbers arising from combinatorial statistics on lattice paths. Journal of Statistical Planning and Inference, 34(1), 35-55.

[2] Reiner, V. (1997). Non-crossing partitions for classical reflection groups. Discrete Mathematics, 177(1-3), 195-222.

[3] Sulanke, R. A. (2000). Counting lattice paths by Narayana polynomials. The Electronic Journal of Combinatorics, 7, R40.

[4] Sloane, N. J. (2008). The on-line encyclopedia of integer sequences. Published electronically at http://oeis.org.

[5] Daboul, S., Mangaldan, J., Spivey, M. Z., \& Taylor, P. J. (2013). The Lah Numbers and the $\mathrm{n}$ th Derivative of $e^{\frac{1}{x}}$. Mathematics Magazine, 86(1), 39-47.

(C) 2020 by the authors; licensee PSRP, Lahore, Pakistan. This article is an open access article distributed under the terms and conditions of the Creative Commons Attribution (CC-BY) license (http://creativecommons.org/licenses/by/4.0/). 